\title{
Appreciation to Multimedia Tools and Applications Reviewers
}

Published online: 9 April 2016

(C) Springer Science+Business Media New York 2016

For helping us deliver timely decisions to our authors, the Editor-in-Chief and Publisher would like to thank the following individuals that contributed reviews between January 1, 2015 and December 31, 2015. We applaud your efforts and dedication to the community.

Hadid Abdenour
Mohammad Abdullah-
AI-Wadud
Velibor Adzic
Reza Afrouzian
Naiara Aginako
Harry Agius
Rajeev Agrawal
Ashfaq Ahmad
Muneer Ahmad
Uzair Ahmad
Dewan Tanvir Ahmed
Mohamed Ahmed
Shohel Ahmed
Zeeshan Ahmed
Hyobeom Ahn
Jaesuk Ahn
Jayson Ahn
Jin-Ho Ahn
Patrick Aichroth
Ali Al-Haj
Hazrat Ali
Rahman Ali
Hend Al-Khalifa
Anas Al-Roubaiey
Ola Amayri
Irene Amerini
Jeong-Won An
Le An

$\begin{array}{ll}\text { Sangjun An } & \text { Changchun Bao } \\ \text { Maria Teresa Andrade } & \text { Lei Bao } \\ \text { Marios Angelides } & \text { Zhenkun Bao } \\ \text { Marco Anisetti } & \text { Lorenzo Baraldi } \\ \text { Marios Anthimopoulos } & \text { Remigiusz Baran } \\ \text { M. Antona } & \text { Vittorio Baroncini } \\ \text { Rinki Arya } & \text { Silvio Barra } \\ \text { Abu Asaduzzaman } & \text { Claude Barras } \\ \text { Akira Asano } & \text { Federico Bartoli } \\ \text { Sinem Aslan } & \text { Santosh Basapur } \\ \text { Pedro Assunção } & \text { Ahmad Hoirul Basori } \\ \text { Hanif Azhar } & \text { Muhammet Bastan } \\ \text { Yogameena B } & \text { Marek Bauer } \\ \text { Noboru Babaguchi } & \text { Luis Baumela } \\ \text { Enrico Baccaglini } & \text { Shahab Bayati } \\ \text { Thierry Badard } & \text { Neslihan Bayramoglu } \\ \text { Claudio Baecchi } & \text { Federico Becattini } \\ \text { Myong-Hyun Baek } & \text { Liu BeiBei } \\ \text { Xiang Bai } & \text { Alexander Bekiarski } \\ \text { Yong Bai } & \text { Anna Belardinelli } \\ \text { Werner Bailer } & \text { Sadok Ben Yahia } \\ \text { Sheikh Tahir Bakhsh } & \text { Yannick Benezeth } \\ \text { Selcuk Baktir } & \text { Jenny Benois-Pineau } \\ \text { Vidhya Balasubramanian } & \text { Stefano Berretti } \\ \text { Sandra Baldassarri } & \text { Marco Bertini } \\ \text { Laercio Baldochi } & \text { Jesus Bescos } \\ \text { Javier Balladini } & \text { Ed Bezerra } \\ \text { Panos Bamidis } & \text { Subhash Bhalla } \\ \text { Hichem Bannour } & \text { Darren Bharanitharan } \\ & \end{array}$


S. Bharathi

Bharat Bhargava

Chidansh Bhatt

Debnath Bhattacharyya

Jarosław Białas

Jingwen Bian

Lubomir F. Bic

Mohsin Bilal

Ioan Marius Bilasco

Milan Bjelica

Lizzy Bleumers

Fernando Boavida

Maya Bojilova

Enrico Bondi

David Bong

Miguel Bordallo Lopez

Monica Bordegoni

Y.L. Borissov

Fernando Boronat

Joseph Bosco

Laszlo Böszörmenyi

Janos Botzheim

Hamdi Jamel Bouchech

Imed Bouchrika

Christos Bouras

V. Michael Bove Jr

Alexia Briassouli

Shuhui Bu

Tobias Bürger

John Burns

Jin Wook Byun

Haibin Cai

Rongtai Cai

Rui Cai

Weidong Cai

Ying Cai

Roberto Caldelli

Jorge Caldera-Serrano

Carmen Camara

Antonio Camarena-Ibarrola

Elena Camossi

Brian Canada

Edgar Cano Cruz

Virgino Cantoni

Fang Cao

Jiangtao Cao

Junjie Cao
Juan Vicente Capella

Hernández

Daniela Cardillo

Franco Alberto Cardillo

Maria Chiara Caschera

Renan Cattelan

Marc Cavazza

Antonio Cedillo-Hernandez

Elena Cerezuela-Escudero

Christophe Cerisara

Pablo Cesar

Xiujuan Chai

Chee Seng Chan

Tommy Yu-Wei Chan

Y.K. Chan

Yu-Wei Chan

Krishna Chandramouli

Chung-Liang Chang

Deokmoon Chang

Hsuan-Ting Chang

Xiaojun Chang

Yao-Tang Chang

Yi-Ying Chang

Delphine Charlet

Richard Chbeir

Xiangjiu Che

Salman Cheema

Bo-Wei Chen

Chao Chen

Chao-Yun Chen

Chen Chen

Chen-Yu Chen

Chia-Chen Chen

Chia-Yen Chen

Ching-Yi Chen

Dan Chen

Fan Chen

Gen-Fang Chen

Hua-Ching Chen

Hua-Wei Chen

Hung-Kuang Chen

Jie Chen

Jing Chen

Jingjijng Chen

Jingying chen

Jinjun Chen

Ju-Chin Chen
Kuan-Hung Chen

Li-Wei Chen

Shengyong Chen

Shi-Ming Chen

Tong Chen

Xiangping Chen

Xiaobo Chen

Xiaojiang Chen

Xiao-jun Chen

Xiaolei Chen

Xiaowu Chen

Xin Chen

Yen-Wei Chen

Yi-Chung Chen

Ying Chen

Yi-Shin Chen

Bo-Chao Cheng

Chao Cheng

Hang Cheng

Jian Cheng

Xian-yi Cheng

Xiaochun Cheng

Cheng-Chieh Chiang

Been-Chian Chien

Pei-Ling Chien

Naveen Chilamkurti

Monica Chis

Wojciech Chmiel

Jacek Chmielewski

Chung Woon Cho

DS Cho

Han-Jin Cho

Jin-Sung Cho

Jonghwan Cho

Moon-Taek Cho

Ok-Hue Cho

Anthony Choi

Changhoon Choi

Jaden Choi

Ka-Cheng Choi

Min Choi

Sangsoo Choi

YongSoo Choi

Yonshik Choi

Wojciech Chojnacki

Ryszard Choraś

Varin Chouvatut 
Carol Chu

HaiCheng Eric Chu

Wei-Ta Chu

Y.-S. Chu

Ching-Song Chuang

Kwang-Sik Chun

Se-Hak Chun

Ronald Chung

Tommy Chung

Stelvio Cimato

Gianluigi Ciocca

António Coelho

Rémi Cogranne

Dinu Coltuc

Nuno Correia

Alceu Ferraz Costa

Mickael Coustaty

Stephen Cox

Rui Cruz

Lin Cui

Minshan Cui

Charlie Cullen

Stuart Cunningham

Marilia Curado

Kevin Curran

Boguslaw Cyganek

Andrzej Czyżewski

Tasos Dagiuklas

Deborah Dahl

Baisheng Dai

Rossana Damiano

Wei Dan

Minh-Son Dao

Gong Daofu

Wang Daoshun

Petros Daras

Sergiu Dascalu

Tiago José de Carvalho

Maria De Marsico

Toon De Pessemier

Marcos del Pozo Baños

Jaime Delgado

Emmanuel Dellandrea

Anne Demoisy

Hepu Deng

Lawrence Y. Deng

Zhengjie Deng
Oscar Deniz Suarez

Pranab Kumar Dhar

Dario Di Fina

Luhong Diao

Fernando Díaz de María

Rebeca Díaz Redondo

C. Dimoulas

Nizamud Din

Ing-Jr Ding

Christos Diou

S. Doa

Grzegorz Dobrowolski

Debi Prosad Dogra

Jiri Dokulil

Mario Döller

Juan Domínguez-Morales

Hongli Dong

Jing Dong

Shi Dong

Xinghui Dong

Anastasios Doulamis

Nikolaos Doulamis

Fadoua Drira

Peijun Du

Qinghe Du

Fuqing Duan

Jean-Claude Dufourd

Arianna D'Ulizia

Hai Duong Trong

Ambar Dutta

Malay Kishore Dutta

Hossein Ebrahimnezhad

Said Ech-Chadi

Wolfgang Effelsberg

Muhammad Ehsan

Younès El Bouzekri El Idrissi

Ahmed El Oualkadi

Georgia Elafoudi

Atilla Elci

Gorka Epelde

Victor Epitropou

Marcos Escudero

Ali Etemad

Ralph Ewerth

Pavlos Fafalios

Mohamed Waleed Fakhr
Fabrizio Falchi

Ben Falchuk

Dengping Fan

Jianping Fan

Xin Fan

Hui Fang

Lanting Fang

Yinfeng Fang

Youhan Fang

Yuming Fang

Lorenzo Favalli

Hsuan-Ming Feng

Hui Feng

Jin Feng

Jufu Feng

Qi Feng

Wei Feng

Yinfu Feng

Gerardo Fernandez Escribano

Andres Fernandez-Revelles

Claudio Ferrari

Anselmo Ferreira

Adriano Fiorese

Francesco Flammini

Martin Fleury

Arthur Flexer

Antonio Foncubierta-

Rodríguez

Simon Fong

David Fouhey

Panayotis Fouliras

Edward Fox

Prior Fred

Dariusz Frejlichowski

Maria Frucci

Weina Fu

Wenxian $\mathrm{Fu}$

Zhenyong $\mathrm{Fu}$

Zhonghua $\mathrm{Fu}$

Zou Fuhao

Marco Furini

Wei Fushan

Davide Gadia

Ombretta Gaggi

Jakub Gałka

Leonardo Galteri

Chenqiang Gao 
Jinlan Gao

Qinghai Gao

Tao Gao

Xianghui Gao

Xinbo Gao

Yue Gao

Yueming Gao

Zan Gao

Alejandro Garcia-Alonso

Alvaro García-Martín

Yun Ge

S. Geetha

Xin Geng

Claudio Gennaro

G. Georgalis

Gheorghita Ghinea

Gabriele Gianini

D. C. Gibbon

Miriam Gil

Gwangyong Gim

Xavier Giro

Adam Gluszek

Abel Gomes

Laurent Gomez

Marta Gomez-Barrero

Liyu Gong

Zhiguo Gong

Adilson Gonzaga

Philippe-Henri Gosselin

Naka Gotoda

Carsten Gottschlich

Rudinei Goularte

Bernard Grabot

Michael Granitzer

Patrizia Grifoni

Jens Grivolla

William Grosky

Xiaoqing $\mathrm{Gu}$

Jiunian Guan

Naiyang Guan

Javier Guerra-Casanova

Hanene Guesmi

Marcelo Guimarães

Stephen Gulliver

Bin Guo

Fan Guo

Huiping Guo
Kehua Guo

Yanwen Guo

Yu Guo

Yuliang Guo

Benjamin Guthier

GuiRyong Ha

Le Thanh Ha

$\mathrm{Nan} \mathrm{Ha}$

James K. Hahn

Md. Abdul Hamid

A. Ben Hamza

Byeong-jun Han

Dongfeng Han

Dong-Guk Han

InnSung Han

Jungong Han

Junwei Han

Kyusuk Han

Nguyen Dinh Han

Sang-Min Han

Weiwei Han

Xiaoran Han

Yahong Han

Allan Hanbury

Guimin Hang

Jingbin Hao

Yongshen Hao

Mohammad Haque

Hadi Harb

Najam ul Hasan

Mohammad Mehedi Hassan

Najam Ul Hassan

David Hästbacka

Fazhi He

Hongjie $\mathrm{He}$

Huang He

Qian $\mathrm{He}$

Ran He

Xuejian $\mathrm{He}$

Hermann Hellwagner

Seok Won Heo

Eelco Herder

Luis Herranz

Daniel Herrera Castro

Helmut Hlavacs

Lau Siong Hoe

Andreas Holzinger
Bong Hwa Hong

Jeong-Mo Hong

Min Hong

Qingyang Hong

SeongSik Hong

Suck-Joo Hong

SungDae Hong

Wenxue Hong

Xiaopeng Hong

Wang Hongdou

Kouh Hoonjoon

Frank Hopfgartner

M. Shamim Hossain

A-Lin $\mathrm{Hou}$

Chunping Hou

Chen Houjin

Chih-Hsien Hsia

Kuei-Fang Hsiao

Chen-Chiung Hsieh

Chi Wen Hsieh

Hui-huang Hsu

Jia-Lien Hsu

Bing $\mathrm{Hu}$

$\mathrm{Min} \mathrm{Hu}$

Qingsong $\mathrm{Hu}$

Tiejun $\mathrm{Hu}$

Wu-Chih $\mathrm{Hu}$

Yongjian $\mathrm{Hu}$

Kien Hua

Kun Hua

Yang Hua

Ruohong Huan

Cheng-Ta Huang

Hsinfu Huang

Jiaoying Huang

Junshi Huang

Liang Huang

Qiang Huang

Qingming Huang

Tianyu Huang

Wei Huang

Xuan Huang

Yan Huang

Yin-Fu Huang

Yonggang Huang

Benoit Huet

Chien-Lun Hung 


\begin{tabular}{|c|c|c|}
\hline Jason C. Hung & Yanfei Jia & Seung-Seok Kang \\
\hline Patrick Hung & Muwei Jian & Shin-Jin Kang \\
\hline Wei Hung & Wu Jianfeng & Sungho Kang \\
\hline Neil Hurley & Bo Jiang & Mohan Kankanhalli \\
\hline Wolfgang Hürst & Ji-Han Jiang & Rajkumar Kannan \\
\hline Sami Huttunen & Junjun Jiang & Jalal Karam \\
\hline Kuo Yuan Hwa & Shaohua Jiang & Bahadir Karasulu \\
\hline Heasoo Hwang & Shuqiang Jiang & Harish Katti \\
\hline Jen-Ing G. Hwang & Wenbin Jiang & Y. S. Kavian \\
\hline Kyu-Baek Hwang & Xiaoyue Jiang & Young Min Kawk \\
\hline Sue-Fen Hwang & Yu-Gang Jiang & Michal Kawulok \\
\hline Wen-Jyi Hwang & Zhiwei Jiang & Eamonn Keogh \\
\hline WonJun Hwang & Lu Jicang & Wu Keyu \\
\hline Roberto Iacoviello & ChangLong Jin & Shehzad Khalid \\
\hline Ichiro Ide & Xiaogang Jin & Abdul Aziz Khan \\
\hline Andrey Ignatov & Xue-bo Jin & Adil Mehmood Khan \\
\hline Hiroaki Ikeda & Yi Jin & Murad Khan \\
\hline Kohei Inoue & Young-geun Jin & Zahid Khan \\
\hline Bogdan Ionescu & Tian Jing & Siamak Khatibi \\
\hline Muhammad Munwar Iqbal & Xuan Jing & Asad Masood Khattak \\
\hline Cesar Isaza & Liu Jiufen & Muazzam A. Khan Khattak \\
\hline Md Saiful Islam & Jun Jo & Duc Kieu \\
\hline Rashedul Islam & Juyeon Jo & Byung-Gyu Kim \\
\hline SK Islam & Seng-Kyoun Jo & Cheong Ghil Kim \\
\hline Ivan Ivanov & Zhaojie Ju & Daehoon Kim \\
\hline Yuji Iwahori & Jozef Juhár & DongHyun Kim \\
\hline Chen J.Z & Jong-Seo Jun & Eui-Jik Kim \\
\hline Maria Letizia Jaccheri & Sang Jun & Euntai Kim \\
\hline Reza Jafari & Sanghoon Jun & Hansung Kim \\
\hline Arfan Jaffar & Hanmin Jung & Heejeong Kim \\
\hline Nuraini Jamil & Hyosook Jung & Hyenchol Kim \\
\hline Sang-dong Jang & In Chul Jung & Hyungshin Kim \\
\hline Young-Chan Jang & Jae-Pil Jung & HyunJin Kim \\
\hline Bartosz Jasiul & Ki-Hyun Jung & Jai-Hoon Kim \\
\hline Jinho Jeon & KyeDong Jung & Jantae Kim \\
\hline Hwa-Young Jeong & Seung-Won Jung & Jeong-Heon Kim \\
\hline Jongmyeon Jeong & Sewook Jung & Jihyung Kim \\
\hline SoHyeon Jeong & Young-Ae Jung & Jin-Mook Kim \\
\hline Taikyeong Jeong & Rene Kaiser & Jong-Hun Kim \\
\hline Wookyeong Jeong & Yannis Kalantidis & Jongman Kim \\
\hline Rongrong Ji & Hari Kalva & Jongsung Kim \\
\hline S. Ji & Carlos Kamienski & Jongweon Kim \\
\hline Wen Ji & Shunsuke Kamijo & Jungsun Kim \\
\hline Xiaofei Ji & Dong-joong Kang & Jungyeon Kim \\
\hline Zhong Ji & Dongwann Kang & Kye Soo Kim \\
\hline Chengcheng Jia & Henry Kang & Mucheol Kim \\
\hline Jia Jia & Li-Wei Kang & Namgi Kim \\
\hline
\end{tabular}


Sohee Kim

Soo-Cheol Kim

Soojin Kim

Soo-Kyun Kim

Sun-Joo Kim

Tae-Hoon Kim

TK Kim

Yanghoon Kim

Yong-Ho Kim

Yongjoo Kim

Young-Gab Kim

Shinichi Kimura

Georgios Kioumourtzis

Tatsuya Kitamura

Ralf Klamma

Jirka Klaue

Hendrik Knoche

Dae-Sik Ko

Christian Kollmitzer

Mandy Kom

Yiannis Kompatsiaris

Dehui Kong

HyungIl Koo

Kwang Ho Kook

Jari Korhonen

Oliver Korn

Paweł Korus

Harald Kosch

Markus Koskela

Hristo Kostadinov

Zbigniew Kotulski

Levente Kovács

Nadia Kozievitch

Josip Krapac

Tomas Krilavičius

Bogdan Księżopolski

Panos Kudumakis

Sushil Kumar

B.T.G.S. Kumara

Anton Kummert

Prayoth Kumsawat

Chia-Hsu Kuo

Chung-Ming Kuo

Ting-Chun Kuo

Yong-Lin Kuo

Sanghoon Kwak

Yoon-Sik Kwak
Goo-Rak Kwon

JH Kwon

Suhn Beom Kwon

Nigel Kyseler

Tai-Hung Lai

Yukun Lai

Rodrigo Laiola Guimarães

Hak-Keung Lam

Kam-Yiu Lam

Patrick Lambert

Guo-Cheng Lan

Congyan Lang

Herve Le Borgne

Byoung Yup Lee

Chang-Ha Lee

Changhoon Lee

Chen-Yu Lee

ChongDeuk Lee

DaeWon Lee

Deok Gyu Lee

Deokgyu Lee

Eunyoung Lee

Gyu Myoung Lee

Hee-Jin Lee

Hong joo Lee

Hosin Lee

Hsiao-Ping Lee

Huang-Chen Lee

Hyokyeong Lee

Jaepil Lee

Jaesung Lee

Jeong Jin Lee

Jihyun Lee

Jinhyoung Lee

John Lee

Jong Kwan Lee

Jongwon Lee

Jong-Yun Lee

Jung-Won Lee

Junwon Lee

Keonsoo Lee

Ki-Hoon Lee

Kilhung Lee

Kye-Shin Lee

Minsoo Lee

Mun-Kyu Lee

Rae-Chun Lee
Sang-Gil Lee

Yang Sun Lee

Yeon-Ok Lee

Youngseok Lee

Yunjin Lee

Frederic Lefebvre

Fabrice Lefèvre

Wang Lei

Miguel Leitão

George Lekakos

Lu Leng

Jin-Jang Leou

Mikołaj Leszczuk

Jenq-Shiou Leu

Carson Leung

Clement Leung

Bailin Li

Bing Li

Bing Nan Li

Bo Li

Demin Li

Fei Li

Fengyong Li

G. L. Li

Gongfa Li

Guiqing Li

Hongyu Li

Jia Li

Junfeng Li

$\mathrm{Li} \mathrm{Li}$

Liang Li

Linde Li

Panchi Li

Ping Li

Qing Li

Shih-An Li

Shiwu Li

Shiying Li

Tao Li

Teng Li

Warchester Li

Weihai Li

Weimin Li

Xiaolong Li

Xin Li

Yantao Li

Yao Li 


\begin{tabular}{|c|c|c|}
\hline Yin Li & Kui Liu & Mathias Lux \\
\hline Yingming Li & Li Liu & Zhihan Lv \\
\hline Yuan Li & Licheng Liu & Fengying Ma \\
\hline Zheng Li & Ligang Liu & Huadong Ma \\
\hline Zhixin Li & Maofu Liu & Kevin Ma \\
\hline Lin Liang & Miao Liu & Lin $\mathrm{Ma}$ \\
\hline Yun Liang & Nan Liu & Yiwei Ma \\
\hline Honghong Liao & Pei-Zhong Liu & Zhigang Ma \\
\hline Pei-Hung Liao & Qiong Liu & Namunu C. Maddage \\
\hline Wen-Hung Liao & Risheng Liu & Owais Mahmudi \\
\hline Xin Liao & ShaoHui Liu & Long Mai \\
\hline Mulugeta Libsie & Shaoying Liu & Luca Mainetti \\
\hline W.-N. Lie & Shigang Liu & Abdul Majid \\
\hline Jongseok Lim & Shuai Liu & Konstantinos Makantasis \\
\hline Kyungsoo Lim & Tianming Liu & Yasushi Makihara \\
\hline Sang Boem Lim & Weifeng Liu & Athanasios Malamos \\
\hline Chang Hong Lin & Weiqiang Liu & Hafiz Malik \\
\hline Chih-Yang Lin & Xing Liu & Thomas Mandl \\
\hline Ching-Chiuan Lin & Xueliang Liu & Marco Manfredi \\
\hline Chunyu Lin & Yang Liu & Jiafa Mao \\
\hline Hao-Chiang Koong Lin & Ye Liu & Tomasz Marciniak \\
\hline ShuJin Lin & Yi Liu & Nenad Markuš \\
\hline Wei-Yang Lin & Yuan-Chen Liu & Oge Marques \\
\hline Xiaofan Lin & Yujie Liu & Manoel Marques Neto \\
\hline Xiaopeng Lin & Zhan Liu & Jose Martinez \\
\hline Yen-Yu Lin & Zhenbao Liu & M Mehedi Masud \\
\hline Yih-Chuan Lin & Silvia Llorente & Liam Mayron \\
\hline Yu-Tzu Lin & Richard K. Lomotey & Giuseppe Mazzola \\
\hline Zijia Lin & Xi Long & Kevin McGuinness \\
\hline Marino Linaje & Xianzhong Long & Rashid Mehmood \\
\hline Georges Linares & John Louise & Zahid Mehmood \\
\hline Hefei Ling & Benny Lövström & Hegeng Mei \\
\hline Giuseppe Lisanti & Huchuan Lu & Britta Meixner \\
\hline Thomas Little & $\mathrm{Ke} \mathrm{Lu}$ & Rufael Mekuria \\
\hline Anan Liu & $\mathrm{Lu} \mathrm{Lu}$ & Fanrong Meng \\
\hline Baolong Liu & Ning Lu & B. Merialdo \\
\hline Bei-bei Liu & Qingmei Lu & Vasileios Mezaris \\
\hline Bo Liu & Yijuan Lu & Qiguang Miao \\
\hline Chanjuan Liu & Zonglei Lu & Zelang Miao \\
\hline Chengming Liu & Zorro Luis & Libor Michalek \\
\hline Damon Shing-Min Liu & Chunbo Luo & Peter Mihajlik \\
\hline Gaowen Liu & Hangzai Luo & Sarah Miller \\
\hline Haiyuan Liu & Jun Luo & Walter Miller \\
\hline Hong Liu & Xiangyang Luo & Gihong Min \\
\hline Huafeng Liu & Yan-Min Luo & Weiqing Min \\
\hline Jin Liu & Yong Luo & Miroslav Minović \\
\hline Jinguo Liu & Zhongxuan Luo & Hemant Misra \\
\hline
\end{tabular}


Masaru Miyazaki

Ahmed Mohammad

Aree Mohammed

Farahnaz Mohanna

Saraju Mohanty

Josep Monguet

Jae Sung Moon

Seok-Jae Moon

Seung Ki Moon

Igor Moraes

Annette Morales-Gonzalez

Jürgen Moßgraber

Jose Mossi

Anastasia Moumtzidou

Konstantinos Moustakas

$\mathrm{Mu} \mathrm{Mu}$

Debora Muchaluat-Saade

Ghulam Muhammad

Khan Muhammad

Snehasis Mukherjee

Philippe Mulhem

Ethan Munson

Moyen Mustaquim

Duygu Mutlu

Karthikeyan Nagarajan

Hiroshi Nakajima

Ryohei Nakatsu

Junghyun Nam

Haiq Nan

Fabio Narducci

Sarwat Nasir

Aziz Nasridinov

Prabhu Natarajan

Andres Adolfo Navarro

Newball

Paolo Nesi

Augusto Neto

Robert Newcomb

Dung Nguyen

Tri Nguyen

Jianjun Ni

Liqiang Nie

S. Nie

Weizhi Nie

Xiushan Nie

Naoko Nitta

Xiamu Niu
Yuzhen Niu

Luis Nonato

Klimis Ntalianis

Marianna Obrist

Seung Joon Oh

Yong $\mathrm{Oh}$

Neil O'Hare

Waleed Dar Sham Oon

Vincent Oria

Irena Orovic

Zongying $\mathrm{Ou}$

Marco Padula

Dra.Carla Pagliari

Pietro Pala

Simone Palazzo

David Palma

Jen-Yi Pan

Rongjiang Pan

Sung Bum Pan

Xunyu Pan

Jose Panach

Costas Panagiotakis

Jeng Pang

Juan Pantrigo

Luca Paolino

George Papagiannakis

Laura Papaleo

Deepti Pappusetty

Adapa Paramkusam

Saurin Parikh

Doosun Park

Heemin Park

Jaehyeoung Park

Joonseok Park

Kang Ryoung Park

Kihong Park

Kinam Park

Sangoh Park

Sangseo Park

Seung-Bo Park

Seung-Jin Park

Taejung Park

Youngho Park

Ignazio Passero

Gilberto Pastorello

Al-Sakib Khan Pathan

Srikanta Patnaik
Jialiang Peng

Limei Peng

Yong Peng

Yuwei Peng

Stefan-Gheorghe Pentiuc

Fernando Pereira

Xavier Perramon

Andrew P. Peterson

Raphael Phan

Somnuk Phon-Amnuaisuk

Antonio Picariello

Massimo Piccardi

Jose Luis Pina

Julien Pinquier

Irena Pletikosa Cvijikj

Matúš Pleva

Moacir Ponti

Marco Porta

Marcelo Porto

Balakrishnan Prabhakaran

Marius Preda

William Puech

Honggang Qi

Lin Qi

Shuhan Qi

Wei Qi

Yinan Qi

Xueming Qian

Yao Qian

Mengyu Qiao

Chuan Qin

Jinlong Qin

Shengfeng Qin

Yuchu Qin

Chunmei Qing

Cheng Qingfeng

Zhang Qinghe

Yanyun Qu

Marco Quartulli

Paula Queluz

Georges Quénot

Tamer Rabie

Bogdan Raducanu

Ramachandra Raghavendra

Md. Mahfuzur Rahman

Mizanur Rahman

Newlin Rajkumar 


\begin{tabular}{|c|c|c|}
\hline Savitha Ramasamy & Isabel Schwende & Dhananjay Singh \\
\hline Yunbo Rao & Ayan Seal & Przemyslaw Slusarczyk \\
\hline Umer Rashid & Monica Sebillo & Brandon Smith \\
\hline Abdur Razzaque & Lorenzo Seidenari & Monique Snoeck \\
\hline Martin Reisslein & Florian Seitner & Carlos Soares Neto \\
\hline Peng Ren & Byung-Kuk Seo & Jaka Sodnik \\
\hline Tongwei Ren & SangHyun Seo & Koohborfardhaghighi \\
\hline Yongjun Ren & Ssang-Hee Seo & Somayeh \\
\hline Taehyun Rhee & Jongwon Seok & Biao Song \\
\hline Seungmin Rho & Tapio Seppanen & Houbing Song \\
\hline Sidra Riaz & Mustafa Sert & Jingkuan Song \\
\hline Stefano Ricciardi & Behzad Shahraray & Minseok Song \\
\hline Daniel Riccio & Feng Shao & Wei Song \\
\hline Christian Riess & Wenze Shao & Yan Song \\
\hline Giuseppe Rizzo & Li Shaomei & Youjin Song \\
\hline Yong Man Ro & Tarun Sharma & Daniel Sourina \\
\hline Werner Robitza & Bo Shen & Medeni Soysal \\
\hline Marco Roccetti & Hualei Shen & Concetto Spampinato \\
\hline Edgar Roman-Rangel & Jialie Shen & Sebastijan Šprager \\
\hline Teresa Romão & Jinrong Shen & Filippo Stanco \\
\hline Catherine Roussey & Minmin Shen & Pavel Stasa \\
\hline Filip Rudziński & Shuhan Shen & Denal S. Sterling \\
\hline Marcela Ruiz & Wen Zhong Shen & Scott P. Sterling \\
\hline Tomasz Ruść & Bin Sheng & Scott Stevens \\
\hline Seung Taek Ryoo & Michael Sheng & Markus Stocker \\
\hline Eun-Seok Ryu & Victor Sheng & Bjoern Stockleben \\
\hline Seungchul Ryu & Jia-Shing Sheu & Nikolai Stoianov \\
\hline Uk-Jae Ryu & Jianping Shi & Domenico Striccoli \\
\hline Jose M. Saavedra & Jinglun Shi & Chung-Ho Su \\
\hline Reza Sabzevari & Ran Shi & $\mathrm{Ke}-\mathrm{Han} \mathrm{Su}$ \\
\hline Vasiliy Sachnev & Zhenghao Shi & Po-Chyi Su \\
\hline Harald Sack & Timothy K. Shih & Ruidan Su \\
\hline Faisal Saeed & Horng Shi-Jinn & Tianyu Su \\
\hline Celso Alberto Saibel Santos & Byeong-Seok Shin & Zhaopin Su \\
\hline Mukesh Saini & DongHwan Shin & Zhixun Su \\
\hline Ovidio Salvetti & Dongwan Shin & Zhuo Su \\
\hline Shadrokh Samavi & Seong-Yoon Shin & Dan Sui \\
\hline Juan Carlos San Miguel & Youngjoo Shin & Chao Sun \\
\hline André Santanchè & Damon Shing-Min Liu & Fuming Sun \\
\hline Emmanuel Sardis & Kimiaki Shirahama & Jiande Sun \\
\hline Shin'ichi Satoh & P. Shivakumara & Jin Sun \\
\hline Riccado Satta & Muhammad Shoaib & Li Sun \\
\hline Bernhard Schandl & Taeshik Shon & Lifeng Sun \\
\hline Ansgar Scherp & Ho-Nien Shou & Ruxia Sun \\
\hline Jörg Schlötterer & Wanneng Shu & Weifeng Sun \\
\hline Pascal Schoettle & Jia-Jane Shuai & Yanfeng Sun \\
\hline Klaus Schöffmann & Wen Si & Yongqing Sun \\
\hline
\end{tabular}


Zhengxing Sun

Hong K. Sung

Yunsick Sung

Nitin Suresh

Tole Sutikno

Piotr Szczuko

Gabor Szücs

Ryszard Tadeusiewicz

György Takács

Masaki Takahashi

Y. Takefuji

Oobo Takenori

Fang Tan

Huachun Tan

Jieqing Tan

Mingkui Tan

Xiangmin Tan

Pichaya Tandayya

Chang Tang

Dalai Tang

Jianliang Tang

Jin Tang

Mingdong Tang

Sheng Tang

Xun Tang

Yandong Tang

Zhenjun Tang

Dapeng Tao

Lu Tao

Anam Tariq

Ashkan Tashk

Wallapak Tavanapong

Kuan Ta-Wen

Joe Tekli

Zhu Teng

Georg Thallinger

Sabu M. Thampi

Barry-John Theobald

Graham Thomas

Bart Thomee

Nguyen Thuy

Feng Tian

Jing Tian

Xin Tian

Xinmei Tian

Zhiqiang Tian

Domonkos Tikk
Dian Tjondronegoro

Manu Tom

Jose Tornos

Victoria Torres

Ruben Tous

Bruno Augusto Travencolo

Carlos Travieso-Gonzalez

Chih-Fong Tsai

Joseph C. Tsai

Ming-Fong Tsai

Min-Jen Tsai

Pei-Chen Tsai

Konstantinos Tserpes

Theodora Tsikrika

Chrisa Tsinaraki

Raylin Tso

Hang Tu

Yicheng Tu

Francesco Turchini

Jia Uddin

Mohammed Uddin

Shyh-Kuang Ueng

Satoshi Ueno

Andreas Uhl

Sana Ullah

Tiberio Uricchio

Thierry Urruty

Marian Ursu

Manuel Urueña

Pedro Valderas

Alexandre Valle de Carvalho

Egon L. van den Broek

Martin Varela

Angel Varela-Vaca

Domonkos Varga

Athanasios V. Vasilakos

Filippo Vella

Joerg Velten

Raviteja Vemulapalli

Roberto Vezzani

Enrico Vezzetti

Antonio Vieira

Veronica Vilaplana

Alexey Vinel

Giuliana Vitiello

Lazaros Vrysis

Zheng Wan
Cheng Wang

Chuen-Ching Wang

Dong Wang

Fanglin Wang

George Wang

Haipeng Wang

Hongxia Wang

Hsuan-Fu Wang

Jia Wang

Jia-Ching Wang

Jie Wang

Jim Jing-Yan Wang

Jinqiao Wang

Liang Wang

Lijuan Wang

Lizhe Wang

Longbiao Wang

Meng Wang

Mengjun Wang

Mingjia Wang

Peng Wang

Qifan Wang

Ran-Zan Wang

Ruiping Wang

Ruomei Wang

Shangfei Wang

Shilin Wang

Shuhui Wang

Te-Hua Wang

Wei Wang

Wenbo Wang

Xiangyu Wang

Xiaofeng Wang

Xiaolong Wang

Xiuying Wang

$\mathrm{Xu}$ Wang

Xue Wang

Yalin Wang

Yang Wang

Yi Wang

Yiming Wang

Yisong Wang

Yongtao Wang

Yuehui Wang

Zhaohong Wang

Zhengyou Wang

Zhenhai Wang 


\begin{tabular}{|c|c|c|}
\hline Zhibo Wang & Qingzhen Xu & Yunfeng Yang \\
\hline Zhiyong Wang & Ran Xu & Yuxiang Yang \\
\hline Zhongyuan Wang & Songhua $\mathrm{Xu}$ & Kim Yanghoon \\
\hline Jakob Wassermann & Yan Xu & Berrin Yanikoglu \\
\hline Daishi Watabe & Yong Xu & Yuzo Yano \\
\hline Jianguo Wei & Zezhong Xu & Heng Yao \\
\hline Jie Wei & Zheng Xu & Dengpan Ye \\
\hline Jingbo Wei & Zhongwen $\mathrm{Xu}$ & Jin Ye \\
\hline Shikui Wei & Zunyi Xu & Wei Ye \\
\hline Zhe Wei & Li Xuesheng & Chia-Hung Yeh \\
\hline He Wen & Toshihiko Yamasaki & Mei-Chen Yeh \\
\hline Hiping Wen & Bin Yan & Chih-Ta Yen \\
\hline Jinming Wen & Chenggang Yan & Neil Yen \\
\hline Miaowen Wen & Diqun Yan & Shwu-Huey Yen \\
\hline Chi-Yao Weng & Lingyu Yan & Sang-Soo Yeo \\
\hline Martin Winter & WeiQi Yan & Chucai Yi \\
\hline Lars Wolf & Yan Yan & Gangman Yi \\
\hline Kok-Seng Wong & Zengqiang Yan & Kang Yi \\
\hline Marcel Worring & Keiji Yanai & Zhang Yifeng \\
\hline Isaac Woungang & Bing Yang & Zhan Yi-Ju \\
\hline Zygmunt Wrobel & Cheng-Hsing Yang & Yangmi Yim \\
\hline Haotian Wu & Cheng-Hsiung Yang & Baocai Yin \\
\hline Hefeng Wu & Chenguang Yang & Chengjiu Yin \\
\hline Jinjian Wu & Chenhui Yang & Tengfei Yin \\
\hline Jiunn-Lin Wu & Chuan-kai Yang & Dongwen Ying \\
\hline Pengcheng Wu & Chunhuang Yang & Shihui Ying \\
\hline Shulei Wu & Fan Yang & Duan Yitao \\
\hline Tin-Yu Wu & Gelan Yang & Michio Yokoyama \\
\hline Xiao Wu & Hengfu Yang & Samule K. Yoo \\
\hline Xiaomeng Wu & Hsuan-Che Yang & Youngjoong Yoo \\
\hline Zhiyong Wu & Huanjia Yang & Changhuai You \\
\hline Zhizheng Wu & James Yang & Lihua You \\
\hline Yong Xia & Jucheng Yang & Shingchern You \\
\hline Wei Xiang & Jun Yang & Sungkwan Youm \\
\hline Yong Xiang & N.C. Yang & Taek-Young Youn \\
\hline Di Xiao & Sean Yang & Chang-Wu Yu \\
\hline Yang Xiao & Wangxian Yang & Hong Yu \\
\hline Li Xiaolong & Wankou Yang & Hui Yu \\
\hline Cong-Hua Xie & Xiaoshan Yang & Jian Yu \\
\hline Wei-song Xie & Xiaoyuan Yang & Jing Yu \\
\hline Cunlu Xu & Xin Yang & Junqing Yu \\
\hline Dan Xu & Yang Yang & $\mathrm{Li} \mathrm{Yu}$ \\
\hline Guandong Xu & Yanjiang Yang & Miao Yu \\
\hline Guangquan Xu & Yi Yang & Shiqi Yu \\
\hline Haiyan Xu & Yoonseok Yang & Shoou-I Yu \\
\hline Jian Xu & You Yang & Shyr-Shen Yu \\
\hline Jin $\mathrm{Xu}$ & Yulian Yang & Yanru Yu \\
\hline
\end{tabular}


Youngjin $\mathrm{Yu}$

Zeyun Yu

Yuan Yuan

Yun-Hao Yuan

Zhaoquan Yuan

Yang Yuanjie

Wu Yue

Lin Yun

Huang Yung Sung

Xenophon Zabulis

Nelson Zagalo

Pietro Zanuttigh

Farzad Zargari

Jaroslav Zdralek

Huanqiang Zeng

Hui Zeng

Jacky Zeng

Kehan Zeng

Ming Zeng

Tao Zeng

Wei Zeng

ZhaoFeng Zeng

Matthias Zeppelzauer

Yongzhao Zhan

Aixin Zhang

Chenggang Zhang

Chi Zhang

Chunjie Zhang

Dandan Zhang

Daqiang Zhang

Dingwen Zhang

Gang Zhang

Guixu Zhang

Hanwang Zhang

Hao Zhang

Honggang Zhang

Jianguang Zhang

Junping Zhang

Kang Zhang

Lefei Zhang

Lei Zhang

Liang Guo Zhang

Lining Zhang

Litao Zhang

Luming Zhang

Peng Zhang

Qian Zhang
Shanshan Zhang

Shanwen Zhang

Su Zhang

Tianzhu Zhang

Wei Zhang

Weiming Zhang

Weinan Zhang

Weizhan Zhang

Xiaoming Zhang

Xinpeng Zhang

$\mathrm{Xu}$ Zhang

Xuanping Zhang

Xueying Zhang

Xuguang Zhang

Ye Zhang

Yicha Zhang

Yifan Zhang

Yimin Zhang

Yingtao Zhang

Yun Zhang

Yungang Zhang

Yunzhou Zhang

Zeroun Zhang

Zhongbao Zhang

Zhongchuan Zhang

Fangming Zhao

Hanli Zhao

Hong Zhao

Jiakun Zhao

Kuo Zhao

Qian Zhao

Qiudong Zhao

Qiushi Zhao

Sicheng Zhao

Tian Zhao

Wan-Lei Zhao

Xuan Zhao

Yanna Zhao

Yong Zhao

Zhe Zhao

Zhigang Zhao

Lu Zhe-Ming

Qingji Zhen

Zonglei Zhen

Chunhou Zheng

Hong Zheng

Lilei Zheng
Minghui Zheng

Pai Zheng

Wenming Zheng

Yongguo Zheng

Zhigao Zheng

Fan Zhong

Shenghua Zhong

Yingji Zhong

Aimin Zhou

Bin Zhou

Bingyin Zhou

Guoxu Zhou

Haibo Zhou

Jing Zhou

Jinxing Zhou

Liang Zhou

Nan Run Zhou

Qingguo Zhou

Ruolin Zhou

Wengang Zhou

Xiaobing Zhou

Xiaokang Zhou

Yu Zhou

Zhiping Zhou

Haijiang Zhu

Jianqing Zhu

Li Zhu

Xiaofeng Zhu

Yadong Zhu

Zhenfeng Zhu

Zhongkui Zhu

Liansheng Zhuang

Yi Zhuang

Roger Zimmermann

Thi Thi Zin

Bartosz Ziółko

Mariusz Ziółko

Kuan-Sheng Zou

Li Xi Zuo 